\title{
BOUNDARY-NONREGULAR FUNCTIONS IN THE DISC ALGEBRA AND IN HOLOMORPHIC LIPSCHITZ SPACES
}

\author{
L. BERNAL-GONZÁLEZ, A. BONILLA, J. LÓPEZ-SALAZAR, \\ AND J.B. SEOANE-SEPÚLVEDA
}

\begin{abstract}
We prove in this paper the existence of dense linear subspaces in the classical holomorphic Lipschitz spaces in the disc all of whose non-null functions are nowhere differentiable at the boundary. Infinitely generated free algebras as well as infinite dimensional Banach spaces consisting of Lipschitz functions enjoying the mentioned property almost everywhere on the boundary are also exhibited. It is also investigated the algebraic size of the family of functions in the disc algebra that either do not preserve Borel sets on the unit circle or possess the Cantor boundary behavior on the disc.
\end{abstract}

\section{Introduction, Notation and Preliminaries}

A real or complex continuous function defined on a real interval $I$ that is nowhere differentiable at $I$ is called a Weierstrass monster. By applying Baire's category theorem, S. Banach and independently S. Marcinkiewicz $[7,39]$ (see also [41, Chapter 11] and [47]) obtained in 1931 that the set of such functions is residual -that is, its complement is of first category-in the space of continuous functions on $I$ endowed with the topology of uniform convergence in compacta. Passing to the complex plane, in this paper we will focus our attention on those continuous nowhere differentiable functions on the unit circle that can be extended holomorphically on the unit disc with lipschitzian properties.

Before going on, let us fix some notation, mostly standard. As usual, the symbols $\mathbb{N}, \mathbb{N}_{0}, \mathbb{Z}, \mathbb{Q}, \mathbb{R}, \mathbb{C}, \mathbb{D}, \overline{\mathbb{D}}$ and $\mathbb{T}$ will denote the set of positive integers, the set $\mathbb{N} \cup\{0\}$, the set of all integers, the field of rational numbers, the real line, the complex plane, the open unit disc $\{z \in \mathbb{C}:|z|<1\}$, the closed unit disc $\{z \in \mathbb{C}:|z| \leq 1\}$ and the unit circle $\{z \in \mathbb{C}:|z|=1\}$, respectively. The symbol $\mathfrak{c}$ will represent the cardinality of the continuum. A domain is a connected nonempty open subset of $\mathbb{C}$. The space of all holomorphic functions on $\mathbb{D}$ is denoted by $H(\mathbb{D})$ and it is endowed with the compact-open topology. The disc algebra is the Banach space $A(\mathbb{D})$

2010 Mathematics Subject Classification. Primary 30H50; Secondary 15A03, 26A16, 26A27, 46E10.

Key words and phrases. disc algebra, nowhere differentiable function, $\alpha$-lipschitzian function, lineability, spaceability, algebrability. 
of all continuous functions $f: \overline{\mathbb{D}} \rightarrow \mathbb{C}$ with $f \in H(\mathbb{D})$, endowed with the supremum norm. If $\alpha \in(0,1]$ and $z_{0} \in A \subset \mathbb{C}$, then a function $f: A \rightarrow \mathbb{C}$ is said to be $\alpha$-lipschitzian (or $\alpha$-hölderian) at $z_{0}$ whenever

$$
\limsup _{z \rightarrow z_{0}} \frac{\left|f(z)-f\left(z_{0}\right)\right|}{\left|z-z_{0}\right|^{\alpha}}<\infty
$$

Moreover, lipschitzian means 1-lipschitzian, while hölderian means $\alpha$-hölderian for some $\alpha$.

Some terminology extracted from the new theory of lineability will be also needed (see $[2,4,5,10,18,20,23,27,28,46]$ for concepts and results). Assume that $\kappa$ is a cardinal number, $X$ is a vector space and $A \subset X$. The subset $A$ is said to be lineable if there is an infinite dimensional vector space $M$ such that $M \backslash\{0\} \subset A$, and $\kappa$-lineable if such an $M$ can be found so as to satisfy $\operatorname{dim}(M)=\kappa$. If, in addition, $X$ is a topological vector space, then the subset $A$ is said to be spaceable in $X$ whenever there is a closed infinite dimensional vector subspace $M$ of $X$ such that $M \backslash\{0\} \subset A$, while $A$ is $\kappa$-dense-lineable in $X$ if there is a dense vector subspace $M$ of $X$ such that $\operatorname{dim}(M)=\kappa$ and $M \backslash\{0\} \subset A$. Now, assume that $X$ is a vector space contained in some linear algebra. Then the subset $A$ is called algebrable if there is an infinitely generated algebra $M$-that is, the cardinality of any system of generators of $M$ is infinite- such that $M \backslash\{0\} \subset A$. Finally, $A$ is said to be strongly $\kappa$-algebrable if there exists an $\kappa$-generated free algebra $M$ with $M \backslash\{0\} \subset A$. Recall that if $X$ is contained in a commutative algebra, then a set $B \subset X$ is a generating set of some free algebra contained in $A$ if and only if for any $N \in \mathbb{N}$, any nonzero polynomial $P$ in $N$ variables without constant term and any distinct $f_{1}, \ldots, f_{N} \in B$, we have $P\left(f_{1}, \ldots, f_{N}\right) \neq 0$ and $P\left(f_{1}, \ldots, f_{N}\right) \in A$.

Starting from Gurariy [31] -who in 1991 proved the lineability of the class of the Weierstrass monsters- a number of mathematicians have investigated and established the diverse degrees of lineability for this class, and even for smaller classes, as for instance for the class of continuous nowhere hölderian functions: see [3,11,12, 26, 32, 34, 43].

By identifying continuous periodic functions on $\mathbb{R}$ with continuous functions on $\mathbb{T}$, it is natural to pass to the complex plane and study both topological and algebraic size of the family of Weierstrass monsters in the disc algebra, that is, of the set of all $f \in A(\mathbb{D})$ such that $\left.f\right|_{\mathbb{T}}$ is nowhere differentiable. The residuality of this set -and even of the smaller one of those $f \in A(\mathbb{D})$ with $\left.f\right|_{\mathbb{T}}$ nowhere hölderian- has been proved by Eskenazis and Makridis [24,25] in 2014 (see also [35]). In [16] the lineability of the class of Weierstrass monsters in the disc algebra is studied. Specifically, it is there proved that the set

$$
N L(\mathbb{T}):=\left\{f \in A(\mathbb{D}):\left.f\right|_{\mathbb{T}} \text { is not lipschitzian at any point of } \mathbb{T}\right\}
$$


and hence the bigger set

$$
N D(\mathbb{T}):=\left\{f \in A(\mathbb{D}):\left.f\right|_{\mathbb{T}} \text { is not differentiable at any point of } \mathbb{T}\right\}
$$

are $\mathfrak{c}$-dense-lineable in $A(\mathbb{D})$. The assertion is reinforced in [14], where it is shown that the set

$$
N H(\mathbb{T}):=\left\{f \in A(\mathbb{D}):\left.f\right|_{\mathbb{T}} \text { is not hölderian at any point of } \mathbb{T}\right\},
$$

which is contained in $N L(\mathbb{T})$, is strongly $\mathfrak{c}$-algebrable and $\mathfrak{c}$-dense-lineable in $A(\mathbb{D})$. Moreover, there exists a closed infinite dimensional Banach space all of whose nonzero members $f$ are in $A(\mathbb{D})$ but $\left.f\right|_{\mathbb{T}}$ is not hölderian at any point of $\mathbb{T}$.

Our aim in this paper is to contribute to complete these results about "holomorphic Weierstrass monsters" in the setting in the disc algebra. Specifically, it will be shown in Section 3 that, for $\alpha \in(0,1)$, the class of $\alpha$ lipschitzian holomorphic functions in $\mathbb{D}$ that are nowhere differentiable at the circle is $\mathfrak{c}$-dense-lineable in the natural topology, so dense-lineable in an optimal sense in terms of dimension. By allowing good behavior on measurenegligible subsets of $\mathbb{T}$, corresponding spaceability and strong algebrability results are also obtained. In a different-but related-order of ideas, we prove in Section 4 the existence of large algebraic structures inside the family of functions in the disc algebra presenting a strange behavior at the boundary, such as Cantor boundary behavior or non-preservation of Borel sets. Section 2 will be devoted to recall a number of auxiliary results about holomorphic functions in the disc and lineability.

\section{Some PRELIMINARY RESUltS}

For background on holomorphic Lipschitz spaces the reader is referred to [22, Chap. 5] and [42, Chap. 8]. Given $\alpha \in(0,1)$, the classical holomorphic Lipschitz space $\Lambda^{\alpha}(\mathbb{D})$ is defined as the set of all analytic functions on $\mathbb{D}$ such that

$$
|f(z)-f(w)| \leq C|z-w|^{\alpha} \quad(z, w \in \mathbb{D}) .
$$

for some constant $C \in(0,+\infty)$ depending on $f$. Such functions are uniformly continuous on $\mathbb{D}$, so they extend continuously on $\overline{\mathbb{D}}$ and, consequently, every space $\Lambda^{\alpha}(\mathbb{D})$ is contained in the disc algebra $A(\mathbb{D})$. Note that from the continuity and the maximum modulus principle, one gets that $f \in \Lambda^{\alpha}(\mathbb{D})$ if and only if $f \in H(\mathbb{D})$ and a Lipschitz condition of order $\alpha$ as in $(2.1)$ is satisfied for $z, w \in \mathbb{T}$. A natural norm on $\Lambda^{\alpha}(\mathbb{D})$ is the one given by

$$
\|f\|_{\alpha}:=|f(0)|+\sup _{\substack{z \neq w \\ z, w \in \mathbb{D}}} \frac{|f(z)-f(w)|}{|z-w|^{\alpha}},
$$

which makes $\Lambda^{\alpha}(\mathbb{D})$ a non separable Banach space with a topology that is finer than the one inherited from $H(\mathbb{D})$.

According to [30, Theorem 1], the following result is at our disposal. 
Theorem 2.1. Let $f$ be an analytic function in $\mathbb{D}$ given by a power series with Hadamard gaps:

$$
f(z)=\sum_{k=0}^{\infty} a_{k} z^{n_{k}},
$$

with $\frac{n_{k+1}}{n_{k}} \geq \lambda>1$ for all $k \in \mathbb{N}$. Then $f \in \Lambda^{\alpha}(\mathbb{D})$ if and only if $a_{k}=O\left(n_{k}^{-\alpha}\right)$ as $k \rightarrow \infty$.

For $0<\alpha \leq 1$, the holomorphic small Lipschitz space of order $\alpha$ is defined to be the class $\Lambda_{\alpha}(\mathbb{D})$ consisting of all functions $f \in \Lambda^{\alpha}(\mathbb{D})$ such that

$$
|f(z)-f(w)|=o\left(|z-w|^{\alpha}\right) \quad(z, w \in \mathbb{D} \text { and } z \rightarrow w) .
$$

It happens that $\Lambda_{\alpha}(\mathbb{D})$ is a closed subspace of $\Lambda^{\alpha}(\mathbb{D})$ under the Lip- $\alpha$-norm $\|\cdot\|_{\alpha}$, so $\left(\Lambda_{\alpha}(\mathbb{D}),\|\cdot\|_{\alpha}\right)$ is also a Banach space. If $0<\alpha<\beta \leq 1$, then we have that

$$
\Lambda^{\beta}(\mathbb{D}) \subset \Lambda_{\alpha}(\mathbb{D}) \subset \Lambda^{\alpha}(\mathbb{D}) \subset A(\mathbb{D}),
$$

all the inclusions being strict and continuous for the respective topologies. Moreover, as a consequence of Mergelyan's approximation theorem in Lip$\alpha$-norm (see [40, p. 205]), we get the next assertion.

Theorem 2.2. For every $\alpha \in(0,1]$ the polynomials form a dense subset of $\Lambda_{\alpha}(\mathbb{D})$ in Lip- $\alpha$-norm. Consequently, $\left(\Lambda_{\alpha}(\mathbb{D}),\|\cdot\|_{\alpha}\right)$ is a separable Banach space.

The following auxiliary assertion is useful in order to obtain dense-lineability from lineability. Its proof can be found in [17, Theorem 2.3] (see also [3, Theorem 2.2 and Remark 2.5] and [2, Section 7.3]).

Theorem 2.3. Assume that $X$ is a metrizable separable topological vector space and that $\kappa$ is an infinite cardinal number. Suppose that $A$ and $B$ are subsets of $X$ such that $A+B \subset A, A \cap B=\varnothing, B \cup\{0\}$ contains a dense vector subspace of $X$, and $A$ is $\kappa$-lineable. Then $A$ is $\kappa$-dense-lineable in $X$.

Note that since the space $X$ is metrizable and separable, its cardinality is $\operatorname{card}(X)=\mathfrak{c}$ and hence $\operatorname{dim}(X) \leq \mathfrak{c}$. Then $\mathfrak{c}$-dense-lineability is the optimal degree of dense-lineability that one can expect for a subset $A \subset X$.

In order to construct large algebras of strange functions, we will employ the next lemma, that is inspired by the method used in [29] based on the superpositions of a fixed function belonging to the considered class with the representatives of some well chosen algebra of functions.

Lemma 2.4. Let $\Omega$ be a nonempty set, $\kappa$ be a cardinal number and $\mathcal{F}$ be a family of functions $\Omega \rightarrow \mathbb{C}$. Assume that there exist an $\kappa$-generated free algebra $\Phi$ consisting of entire functions and a function $f: \Omega \rightarrow \mathbb{C}$ satisfying that $f(\Omega)$ has an accumulation point in $\mathbb{C}$ and $\varphi \circ f \in \mathcal{F}$ for every $\varphi \in \Phi \backslash\{0\}$. Then $\mathcal{F}$ is strongly $\kappa$-algebrable. 
Proof. Consider the set $\mathcal{A}:=\{\varphi \circ f: \varphi \in \Phi\}$, that is an algebra contained in $\mathcal{F} \cup\{0\}$. Assume that $\mathcal{B}$ is a free generator system for $\Phi$ with $\operatorname{card}(\mathcal{B})=\kappa$. Trivially, $\widetilde{\mathcal{B}}:=\{\varphi \circ f: \varphi \in \mathcal{B}\}$ is a generator system for $\mathcal{A}$. Moreover, $\operatorname{card}(\widetilde{\mathcal{B}})=\kappa=\operatorname{card}(\mathcal{B})$ because $\varphi \circ f \neq \psi \circ f$ as soon as $\varphi \neq \psi(\varphi, \psi \in \Phi)$. Indeed, if $\varphi \circ f=\psi \circ f$, then $\varphi=\psi$ on the set $f(\Omega)$, which has an accumulation point in $\mathbb{C}$, and so $\varphi=\psi$ on the whole $\mathbb{C}$ by the Identity Principle for analytic functions. Finally, $\widetilde{\mathcal{B}}$ is a free generator system. To prove this, assume that $N \in \mathbb{N}$, that $P$ is a polynomial of $N$ complex variables and that $\widetilde{\varphi_{1}}, \ldots, \widetilde{\varphi_{N}}$ are distinct functions in $\widetilde{\mathcal{B}}$ satisfying $P\left(\widetilde{\varphi_{1}}, \ldots, \widetilde{\varphi_{N}}\right)=0$ on $\Omega$. There are mutually different functions $\varphi_{1}, \ldots, \varphi_{N} \in \mathcal{B}$ with $\widetilde{\varphi}_{i}=\varphi_{i} \circ f(i=1, \ldots, N)$. Therefore, $Q \circ f=0$ on $\Omega$, where $Q:=P\left(\varphi_{1}, \ldots, \varphi_{N}\right)$. It follows that $Q=0$ on $f(\Omega)$. Again, the Identity Principle tells us that $Q=0$ on $\mathbb{C}$, which implies $P=0$ because $\mathcal{B}$ is algebraically free. Thus, $\mathcal{A}$ is an $\kappa$-generated free algebra, which concludes the proof.

Remark 2.5. An explicit example in which the technique of the last lemma is applied with $\kappa=\mathfrak{c}$ is provided in [1], where $\Phi$ is the algebra generated by a family of entire functions $\left\{f_{\sigma}\right\}_{\sigma \in(0,1)}$ such that the exponential growth order of $f_{\sigma}$ equals $\sigma$. Another example is given in [6, Proposition 7] (see also [9]), where $\Phi$ is chosen to be -in the complex version given in [13]- the algebra generated by the entire functions $e^{c z}(c \in H)$, where $H \subset(0,+\infty)$ is a linearly $\mathbb{Q}$-independent set.

\section{WEIERSTRASS MONSTERS IN THE CLASSICAL HOLOMORPHIC LIPSCHITZ SPACES}

In this section, we shall be able to find a rich algebraic structure inside the subset of $\Lambda_{\alpha}(\mathbb{D})$ given by

$$
\Lambda_{\alpha} N D(\mathbb{T}):=\left\{f \in \Lambda_{\alpha}(\mathbb{D}):\left.f\right|_{\mathbb{T}} \text { is not differentiable at any point of } \mathbb{T}\right\},
$$

where $\alpha \in(0,1)$. Let $\Lambda_{\alpha} N L(\mathbb{T})$ denote the (smaller) class of functions $f$ in $\Lambda_{\alpha}(\mathbb{D})$ such that $\left.f\right|_{\mathbb{T}}$ is nowhere Lipschitz, that is,

$$
\Lambda_{\alpha} N L(\mathbb{T}):=\left\{f \in \Lambda_{\alpha}(\mathbb{D}): \limsup _{z \in \mathbb{T}, z \rightarrow z_{0}}\left|\frac{f(z)-f\left(z_{0}\right)}{z-z_{0}}\right|=+\infty \text { for every } z_{0} \in \mathbb{T}\right\} .
$$

The next two theorems assert lineability and dense-lineability for these sets in an optimal degree.

Theorem 3.1. For every $\alpha \in(0,1)$, the set $\Lambda_{\alpha} N L(\mathbb{T})$ is $\mathfrak{c}$-lineable. Hence $\Lambda_{\alpha} N D(\mathbb{T})$ is also $\mathfrak{c}$-lineable.

Proof. Let us fix any $\beta \in(\alpha, 1)$, as well as any odd integer $b$ such that $b^{1-\beta}>1+\frac{3 \pi}{2}$. Then

$$
b^{-\beta}>b^{-1}\left(1+\frac{3 \pi}{2}\right) .
$$


Hence, there exists a positive number $c$ such that

$$
b^{-\beta}>c>b^{-1}\left(1+\frac{3 \pi}{2}\right) .
$$

Then

$$
b c>1+\frac{3 \pi}{2} \quad \text { and } \quad b^{-1}<c<b^{-\beta} .
$$

We define

$$
M:=\operatorname{span}\left\{f_{a}(z)=\sum_{n=0}^{\infty} a^{n} z^{b^{n}}: c<a<b^{-\beta}\right\} .
$$

If $c<a<b^{-\beta}$, then $a b^{\beta}<1$ and so $a^{n}=O\left(\left(b^{n}\right)^{-\beta}\right)$. By Theorem 2.1 we obtain that $M$ is a vector subspace of $\Lambda^{\beta}(\mathbb{D})$, which in turn is contained in $\Lambda_{\alpha}(\mathbb{D})$. That is, $M \subset \Lambda_{\alpha}(\mathbb{D})$.

We will prove that the set $\left\{f_{a}: c<a<b^{-\beta}\right\}$ is linearly independent. Let us suppose that $\lambda_{1} f_{a_{1}}+\lambda_{2} f_{a_{2}}+\cdots+\lambda_{k} f_{a_{k}}=0$ for some $k \in \mathbb{N}$, $c<a_{k}<\cdots<a_{2}<a_{1}<b^{-\beta}$ and $\lambda_{1}, \ldots, \lambda_{k} \in \mathbb{C}$. Then

$$
\sum_{n=0}^{\infty}\left(\lambda_{1} a_{1}^{n}+\lambda_{2} a_{2}^{n}+\cdots+\lambda_{k} a_{k}^{n}\right) z^{b^{n}}=0
$$

for every $z \in \overline{\mathbb{D}}$, so the uniqueness of coefficients of the Taylor series yields $\lambda_{1} a_{1}^{n}+\lambda_{2} a_{2}^{n}+\cdots+\lambda_{k} a_{k}^{n}=0$ for every $n \in \mathbb{N}_{0}$. For $n=0, \ldots, k-1$, we obtain a set of $k$ conditions given by the following system:

$$
\left(\begin{array}{cccc}
1 & 1 & \cdots & 1 \\
a_{1} & a_{2} & \cdots & a_{k} \\
\vdots & \vdots & & \vdots \\
a_{1}^{k-1} & a_{2}^{k-1} & \cdots & a_{k}^{k-1}
\end{array}\right)\left(\begin{array}{c}
\lambda_{1} \\
\lambda_{2} \\
\vdots \\
\lambda_{k}
\end{array}\right)=\left(\begin{array}{c}
0 \\
0 \\
\vdots \\
0
\end{array}\right) .
$$

The previous matrix is a Vandermonde matrix, so its determinant is not 0 because $a_{1}, \ldots, a_{k}$ are all different. Therefore, $\lambda_{1}=\lambda_{2}=\cdots=\lambda_{k}=0$. This proves that the set $\left\{f_{a}: c<a<b^{-\beta}\right\}$ is linearly independent and thus $\operatorname{dim}(M)=\mathfrak{c}$.

We will prove that $M \backslash\{0\} \subset \Lambda_{\alpha} N L(\mathbb{T})$. If $f \in M \backslash\{0\}$, then there are $k \in \mathbb{N}, c<a_{k}<\cdots<a_{2}<a_{1}<b^{-\beta}$ and $\lambda_{1}, \ldots, \lambda_{k} \in \mathbb{C} \backslash\{0\}$ such that $f=\lambda_{1} f_{a_{1}}+\cdots+\lambda_{k} f_{a_{k}}$. We may assume $\lambda_{1}=1$ because $\Lambda_{\alpha} N L(\mathbb{T})$ is invariant under scaling.

Let $z_{0}$ be any fixed point in $\mathbb{T}$ and let $x_{0} \in \mathbb{R}$ such that $z_{0}=e^{i \pi x_{0}}$. For each $m \in \mathbb{N}$ there is $s_{m} \in \mathbb{Z}$ such that $b^{m} x_{0}-s_{m} \in(-1 / 2,1 / 2]$. We define

$$
t_{m}:=b^{m} x_{0}-s_{m} \in(-1 / 2,1 / 2] \quad \text { and } \quad x_{m}:=\frac{s_{m}-1}{b^{m}} .
$$

Then $x_{m}=x_{0}-\frac{1+t_{m}}{b^{m}} \rightarrow x_{0}$ as $m \rightarrow \infty$, so $\lim _{m \rightarrow \infty} e^{i \pi x_{m}}=z_{0}$. 
For each $j \in\{1, \ldots, k\}$ we consider the function $u_{j}: \mathbb{R} \rightarrow \mathbb{R}$ defined as

$$
u_{j}(x)=\operatorname{Re}\left[f_{a_{j}}\left(e^{i \pi x}\right)\right]=\sum_{n=0}^{\infty} a_{j}^{n} \cos \left(b^{n} \pi x\right) .
$$

Then

$$
\frac{u_{j}\left(x_{m}\right)-u_{j}\left(x_{0}\right)}{x_{m}-x_{0}}=\sum_{n=0}^{\infty} a_{j}^{n} \frac{\cos \left(b^{n} \pi x_{m}\right)-\cos \left(b^{n} \pi x_{0}\right)}{x_{m}-x_{0}}=S_{j, m}+T_{j, m},
$$

where

$$
S_{j, m}:=\sum_{n=0}^{m-1} a_{j}^{n} \frac{\cos \left(b^{n} \pi x_{m}\right)-\cos \left(b^{n} \pi x_{0}\right)}{x_{m}-x_{0}}
$$

and

$$
T_{j, m}:=\sum_{n=0}^{\infty} a_{j}^{m+n} \frac{\cos \left(b^{m+n} \pi x_{m}\right)-\cos \left(b^{m+n} \pi x_{0}\right)}{x_{m}-x_{0}} .
$$

Now, using the relationship $\cos (x+y)-\cos (x-y)=-2 \sin x \sin y(x, y \in$ $\mathbb{R})$, we derive that

$$
\begin{aligned}
\left|S_{j, m}\right| & =\left|\sum_{n=0}^{m-1} a_{j}^{n} \frac{-2 \sin \left(\frac{b^{n} \pi x_{m}+b^{n} \pi x_{0}}{2}\right) \sin \left(\frac{b^{n} \pi x_{m}-b^{n} \pi x_{0}}{2}\right)}{x_{m}-x_{0}}\right| \\
& \leq \sum_{n=0}^{m-1} \pi\left(b a_{j}\right)^{n}\left|\frac{\sin \left(b^{n} \pi \frac{x_{m}-x_{0}}{2}\right)}{b^{n} \pi \frac{x_{m}-x_{0}}{2}}\right| .
\end{aligned}
$$

Since $\left|\frac{\sin x}{x}\right| \leq 1$ for every $x \in \mathbb{R}$ and $a_{j}>c$, we obtain

$$
\left|S_{j, m}\right| \leq \sum_{n=0}^{m-1} \pi\left(b a_{j}\right)^{n}=\pi \frac{\left(b a_{j}\right)^{m}-1}{b a_{j}-1} \leq \frac{\pi}{b c-1}\left(b a_{j}\right)^{m} .
$$

Note that since $b, s_{m} \in \mathbb{Z}$ and $b$ is odd, we have

$$
\cos \left(b^{m+n} \pi x_{m}\right)=\cos \left(b^{m+n} \pi \frac{s_{m}-1}{b^{m}}\right)=\cos \left(b^{n}\left(s_{m}-1\right) \pi\right)=-(-1)^{s_{m}}
$$

and

$$
\begin{aligned}
\cos \left(b^{m+n} \pi x_{0}\right) & =\cos \left(b^{m+n} \pi \frac{s_{m}+t_{m}}{b^{m}}\right)=\cos \left(b^{n} \pi s_{m}+b^{n} \pi t_{m}\right) \\
& =\cos \left(b^{n} \pi s_{m}\right) \cos \left(b^{n} \pi t_{m}\right)-\sin \left(b^{n} \pi s_{m}\right) \sin \left(b^{n} \pi t_{m}\right) \\
& =(-1)^{s_{m}} \cos \left(b^{n} \pi t_{m}\right) .
\end{aligned}
$$

Therefore, we get

$$
\begin{aligned}
T_{j, m} & =\sum_{n=0}^{\infty} a_{j}^{m+n} \frac{-(-1)^{s_{m}}-(-1)^{s_{m}} \cos \left(b^{n} \pi t_{m}\right)}{-\frac{1+t_{m}}{b^{m}}} \\
& =(-1)^{s_{m}}\left(b a_{j}\right)^{m} \sum_{n=0}^{\infty} a_{j}^{n} \frac{1+\cos \left(b^{n} \pi t_{m}\right)}{1+t_{m}} .
\end{aligned}
$$


Now, note that $a_{j}^{n} \cdot \frac{1+\cos \left(b^{n} \pi t_{m}\right)}{1+t_{m}} \geq 0$ for every $n, m, j$ and that $\cos \left(\pi t_{m}\right) \geq 0$ because $t_{m} \in(-1 / 2,1 / 2]$, so

$$
\left|T_{j, m}\right| \geq\left(b a_{j}\right)^{m} \cdot a_{j}^{0} \cdot \frac{1+\cos \left(b^{0} \pi t_{m}\right)}{1+t_{m}} \geq\left(b a_{j}\right)^{m} \frac{1}{1+\frac{1}{2}}=\frac{2}{3}\left(b a_{j}\right)^{m} .
$$

Moreover, since $a_{j}<b^{-\beta}$, we have

$$
\begin{aligned}
\left|T_{j, m}\right| & =\left(b a_{j}\right)^{m} \sum_{n=0}^{\infty} a_{j}^{n} \frac{1+\cos \left(b^{n} \pi t_{m}\right)}{1+t_{m}} \\
& \leq\left(b a_{j}\right)^{m} \sum_{n=0}^{\infty}\left(b^{-\beta}\right)^{n} \frac{2}{1-\frac{1}{2}}=\frac{4\left(b a_{j}\right)^{m}}{1-b^{-\beta}} .
\end{aligned}
$$

Consequently, we are led to

$$
\frac{2}{3}\left(b a_{j}\right)^{m} \leq\left|T_{j, m}\right| \leq \frac{4}{1-b^{-\beta}}\left(b a_{j}\right)^{m} .
$$

Therefore, by (3.1) and (3.2), we obtain

$$
\begin{aligned}
\left|\frac{u_{j}\left(x_{m}\right)-u_{j}\left(x_{0}\right)}{x_{m}-x_{0}}\right| & =\left|S_{j, m}+T_{j, m}\right| \leq \frac{\pi}{b c-1}\left(b a_{j}\right)^{m}+\frac{4}{1-b^{-\beta}}\left(b a_{j}\right)^{m} \\
& =\left(\frac{\pi}{b c-1}+\frac{4}{1-b^{-\beta}}\right)\left(b a_{j}\right)^{m}
\end{aligned}
$$

and

$$
\begin{aligned}
\left|\frac{u_{j}\left(x_{m}\right)-u_{j}\left(x_{0}\right)}{x_{m}-x_{0}}\right| & \geq\left|T_{j, m}\right|-\left|S_{j, m}\right| \geq \frac{2}{3}\left(b a_{j}\right)^{m}-\frac{\pi}{b c-1}\left(b a_{j}\right)^{m} \\
& =\frac{2\left(b c-1-\frac{3 \pi}{2}\right)}{3(b c-1)}\left(b a_{j}\right)^{m} .
\end{aligned}
$$

If we define

$$
d=\frac{\pi}{b c-1}+\frac{4}{1-b^{-\beta}}>0, \quad e=\frac{2\left(b c-1-\frac{3 \pi}{2}\right)}{3(b c-1)}>0,
$$

then we get

$$
e\left(b a_{j}\right)^{m} \leq\left|\frac{u_{j}\left(x_{m}\right)-u_{j}\left(x_{0}\right)}{x_{m}-x_{0}}\right| \leq d\left(b a_{j}\right)^{m} .
$$

Next, we study the function $v_{j}: \mathbb{R} \rightarrow \mathbb{R}$ defined as

$$
v_{j}(x)=\operatorname{Im}\left[f_{a_{j}}\left(e^{i \pi x}\right)\right]=\sum_{n=0}^{\infty} a_{j}^{n} \sin \left(b^{n} \pi x\right) .
$$

Observe that

$$
\frac{v_{j}\left(x_{m}\right)-v_{j}\left(x_{0}\right)}{x_{m}-x_{0}}=\sum_{n=0}^{\infty} a_{j}^{n} \frac{\sin \left(b^{n} \pi x_{m}\right)-\sin \left(b^{n} \pi x_{0}\right)}{x_{m}-x_{0}}=S_{j, m}^{\prime}+T_{j, m}^{\prime},
$$


where

$$
S_{j, m}^{\prime}:=\sum_{n=0}^{m-1} a_{j}^{n} \frac{\sin \left(b^{n} \pi x_{m}\right)-\sin \left(b^{n} \pi x_{0}\right)}{x_{m}-x_{0}}
$$

and

$$
T_{j, m}^{\prime}:=\sum_{n=0}^{\infty} a_{j}^{m+n} \frac{\sin \left(b^{m+n} \pi x_{m}\right)-\sin \left(b^{m+n} \pi x_{0}\right)}{x_{m}-x_{0}} .
$$

By using the relationship $\sin (x+y)-\sin (x-y)=2 \cos x \sin y(x, y \in \mathbb{R})$, we can estimate

$$
\begin{aligned}
\left|S_{j, m}^{\prime}\right| & =\left|\sum_{n=0}^{m-1} a_{j}^{n} \frac{2 \cos \left(\frac{b^{n} \pi x_{m}+b^{n} \pi x_{0}}{2}\right) \sin \left(\frac{b^{n} \pi x_{m}-b^{n} \pi x_{0}}{2}\right)}{x_{m}-x_{0}}\right| \\
& \leq \sum_{n=0}^{m-1} \pi\left(b a_{j}\right)^{n}\left|\frac{\sin \left(b^{n} \pi \frac{x_{m}-x_{0}}{2}\right)}{b^{n} \pi \frac{x_{m}-x_{0}}{2}}\right| .
\end{aligned}
$$

Since $\left|\frac{\sin x}{x}\right| \leq 1$ for every $x \in \mathbb{R}$ and $a_{j}>c$, we obtain

$$
\left|S_{j, m}^{\prime}\right| \leq \sum_{n=0}^{m-1} \pi\left(b a_{j}\right)^{n}=\pi \frac{\left(b a_{j}\right)^{m}-1}{b a_{j}-1}<\frac{\pi}{b c-1}\left(b a_{j}\right)^{m} .
$$

Now, we consider the series $T_{j, m}^{\prime}$. Since $b, s_{m} \in \mathbb{Z}$, we have on the one hand that

$$
\sin \left(b^{m+n} \pi x_{m}\right)=\sin \left(b^{m+n} \pi \frac{s_{m}-1}{b^{m}}\right)=\sin \left(b^{n} \pi\left(s_{m}-1\right)\right)=0 .
$$

On the other hand, since $b$ is odd,

$$
\begin{aligned}
\sin \left(b^{m+n} \pi x_{0}\right) & =\sin \left(b^{m+n} \pi \frac{s_{m}+t_{m}}{b^{m}}\right)=\sin \left(b^{n} \pi s_{m}+b^{n} \pi t_{m}\right) \\
& =\sin \left(b^{n} \pi s_{m}\right) \cos \left(b^{n} \pi t_{m}\right)+\cos \left(b^{n} \pi s_{m}\right) \sin \left(b^{n} \pi t_{m}\right) \\
& =(-1)^{s_{m}} \sin \left(b^{n} \pi t_{m}\right) .
\end{aligned}
$$

Therefore

$$
T_{j, m}^{\prime}=\sum_{n=0}^{\infty} a_{j}^{m+n} \frac{-(-1)^{s_{m}} \sin \left(b^{n} \pi t_{m}\right)}{-\frac{1+t_{m}}{b^{m}}}=(-1)^{s_{m}}\left(b a_{j}\right)^{m} \sum_{n=0}^{\infty} a_{j}^{n} \frac{\sin \left(b^{n} \pi t_{m}\right)}{1+t_{m}} .
$$

Since $a_{j}<b^{-\beta}$ and $t_{m} \in(-1 / 2,1 / 2]$, we obtain

$$
\left|T_{j, m}^{\prime}\right| \leq\left(b a_{j}\right)^{m} \sum_{n=0}^{\infty}\left(b^{-\beta}\right)^{n} \frac{1}{1-\frac{1}{2}}=\frac{2}{1-b^{-\beta}}\left(b a_{j}\right)^{m} .
$$

If we define

$$
h=\frac{\pi}{b c-1}+\frac{2}{1-b^{-\beta}}>0,
$$

then it follows from (3.4) and (3.5) that, for every $j \in\{1, \ldots, k\}$,

$$
\left|\frac{v_{j}\left(x_{m}\right)-v_{j}\left(x_{0}\right)}{x_{m}-x_{0}}\right|=\left|S_{j, m}^{\prime}+T_{j, m}^{\prime}\right| \leq h\left(b a_{j}\right)^{m} \text {. }
$$


We recall that $f=f_{a_{1}}+\lambda_{2} f_{a_{2}}+\cdots+\lambda_{k} f_{a_{k}}$, where $c<a_{k}<\cdots<a_{2}<$ $a_{1}<b^{-\beta}$ and $\lambda_{2}, \ldots, \lambda_{k} \in \mathbb{C} \backslash\{0\}$. For each $j=2, \ldots, k$, let $p_{j}, q_{j} \in \mathbb{R}$ be such that $\lambda_{j}=p_{j}+i q_{j}$. Then

$$
f\left(e^{i \pi x}\right)=u_{1}(x)+i v_{1}(x)+\sum_{j=2}^{k}\left(p_{j}+i q_{j}\right)\left(u_{j}(x)+i v_{j}(x)\right) .
$$

Hence

$$
\begin{aligned}
\operatorname{Re} \frac{f\left(e^{i \pi x_{m}}\right)-f\left(e^{i \pi x_{0}}\right)}{x_{m}-x_{0}}= & \frac{u_{1}\left(x_{m}\right)-u_{1}\left(x_{0}\right)}{x_{m}-x_{0}}+\sum_{j=2}^{k} p_{j} \frac{u_{j}\left(x_{m}\right)-u_{j}\left(x_{0}\right)}{x_{m}-x_{0}} \\
& -\sum_{j=2}^{k} q_{j} \frac{v_{j}\left(x_{m}\right)-v_{j}\left(x_{0}\right)}{x_{m}-x_{0}} .
\end{aligned}
$$

It follows from (3.3) and (3.6) that

$$
\begin{gathered}
\left|\frac{f\left(e^{i \pi x_{m}}\right)-f\left(e^{i \pi x_{0}}\right)}{x_{m}-x_{0}}\right| \geq\left|\operatorname{Re} \frac{f\left(e^{i \pi x_{m}}\right)-f\left(e^{i \pi x_{0}}\right)}{x_{m}-x_{0}}\right| \\
\geq\left|\frac{u_{1}\left(x_{m}\right)-u_{1}\left(x_{0}\right)}{x_{m}-x_{0}}\right|-\sum_{j=2}^{k}\left|p_{j} \frac{u_{j}\left(x_{m}\right)-u_{j}\left(x_{0}\right)}{x_{m}-x_{0}}\right|-\sum_{j=2}^{k}\left|q_{j} \frac{v_{j}\left(x_{m}\right)-v_{j}\left(x_{0}\right)}{x_{m}-x_{0}}\right| \\
\geq e\left(b a_{1}\right)^{m}-\sum_{j=2}^{k} d\left(b a_{j}\right)^{m}\left|p_{j}\right|-\sum_{j=2}^{k} h\left(b a_{j}\right)^{m}\left|q_{j}\right| \\
=\left(b a_{1}\right)^{m}\left[e-\sum_{j=2}^{k} d\left|p_{j}\right|\left(\frac{a_{j}}{a_{1}}\right)^{m}-\sum_{j=2}^{k} h\left|q_{j}\right|\left(\frac{a_{j}}{a_{1}}\right)^{m}\right] .
\end{gathered}
$$

Finally, since $0<a_{j} / a_{1}<1$ for each $j=2, \ldots, k$ and $b a_{1}>b c>1$, we deduce that

$$
\lim _{m \rightarrow \infty}\left|\frac{f\left(e^{i \pi x_{m}}\right)-f\left(e^{i \pi x_{0}}\right)}{x_{m}-x_{0}}\right|=+\infty
$$

Since

$$
\lim _{m \rightarrow \infty} \frac{x_{m}-x_{0}}{e^{i \pi x_{m}}-e^{i \pi x_{0}}}=\frac{1}{i \pi e^{i \pi x_{0}}} \neq 0,
$$

we obtain

$$
\begin{gathered}
\lim _{m \rightarrow \infty}\left|\frac{f\left(e^{i \pi x_{m}}\right)-f\left(z_{0}\right)}{e^{i \pi x_{m}}-z_{0}}\right|= \\
=\lim _{m \rightarrow \infty}\left|\frac{x_{m}-x_{0}}{e^{i \pi x_{m}}-e^{i \pi x_{0}}}\right| \cdot\left|\frac{f\left(e^{i \pi x_{m}}\right)-f\left(e^{i \pi x_{0}}\right)}{x_{m}-x_{0}}\right|=+\infty .
\end{gathered}
$$

This implies that $f$ is not lipschitzian at $z_{0}$, that is, $f \in M$. Thus, we have shown that $M \backslash\{0\} \subset \Lambda_{\alpha} N L(\mathbb{T})$ and the proof is concluded. 
Remark 3.2. The last theorem makes no sense for the case $\alpha=1$, because every $\Lambda^{1}$-function is 1-lipchitzian on $\mathbb{T}$. Even the set $\left\{f \in \Lambda^{1}(\mathbb{D}): f\right.$ is nowhere differentiable on $\mathbb{T}\}$ is empty, because any function of bounded variation on a real interval is differentiable almost everywhere [48, p. 32].

Theorem 3.3. Let $\alpha \in(0,1)$. Then the set $\Lambda_{\alpha} N L(\mathbb{T})$ is $\mathfrak{c}$-dense-lineable in $\Lambda_{\alpha}(\mathbb{D})$. Hence $\Lambda_{\alpha} N D(\mathbb{T})$ is also $\mathfrak{c}$-dense-lineable in $\Lambda_{\alpha}(\mathbb{D})$.

Proof. Let $\mathcal{P}$ denote the set of restrictions to $\overline{\mathbb{D}}$ of the family of all polynomials on $\mathbb{C}$. By Mergelyan's theorem in Lip- $\alpha$-norm (Theorem 2.2), $\Lambda_{\alpha}(\mathbb{D})$ is a separable Banach space with the Lip- $\alpha$-norm and $\mathcal{P}$ is a dense vector subspace of $\Lambda_{\alpha}(\mathbb{D})$. Moreover, if $g \in \Lambda_{\alpha} N L(\mathbb{T}), P \in \mathcal{P}$ and $z_{0} \in \mathbb{T}$, then there exists a sequence $\left(z_{m}\right)_{m=1}^{\infty}$ in $\mathbb{T}$ such that

$$
\lim _{m \rightarrow \infty}\left|\frac{g\left(z_{m}\right)-g\left(z_{0}\right)}{z_{m}-z_{0}}\right|=+\infty .
$$

Then

$$
\begin{aligned}
& \lim _{m \rightarrow \infty}\left|\frac{(g+P)\left(z_{m}\right)-(g+P)\left(z_{0}\right)}{z_{m}-z_{0}}\right| \geq \\
\geq & \lim _{m \rightarrow \infty}\left|\frac{g\left(z_{m}\right)-g\left(z_{0}\right)}{z_{m}-z_{0}}\right|-\left|P^{\prime}\left(z_{0}\right)\right|=+\infty .
\end{aligned}
$$

Hence $g+P$ is not lipschitzian at $z_{0}$. This proves that $g+P \in \Lambda_{\alpha} N L(\mathbb{T})$, that is, $\Lambda_{\alpha} N L(\mathbb{T})+\mathcal{P} \subset \Lambda_{\alpha} N L(\mathbb{T})$. Moreover, of course, $\Lambda_{\alpha} N L(\mathbb{T}) \cap \mathcal{P}=\varnothing$. It follows from Theorem 2.3 (with $X=\Lambda_{\alpha}(\mathbb{D}), A=\Lambda_{\alpha} N L(\mathbb{T}), B=\mathcal{P}$ and $\kappa=\mathfrak{c})$ that $\Lambda_{\alpha} N L(\mathbb{T})$ is $\mathfrak{c}$-dense-lineable in $\Lambda_{\alpha}(\mathbb{D})$.

Concerning the existence of large closed subspaces, we may obtain some kind of spaceability if we relax the condition of "not lipschtzian at any point of the circle" up to "not lipschitzian at almost everywhere on the circle". Here "almost everywhere" (a.e.) is understood with respect to the Lebesgue measure $\frac{d \theta}{2 \pi}$ on $\mathbb{T}$ and the full-measure subset of $\mathbb{T}$ such that $f$ is not lipschitzian at every point of it may depend on $f$. For every $\alpha \in(0,1)$, we denote

$$
\Lambda_{\alpha} N L_{\mathrm{ae}}(\mathbb{T}):=\left\{f \in \Lambda_{\alpha}(\mathbb{D}): f \text { is not lipchitzian a.e. on } \mathbb{T}\right\}
$$

Spaceability for this set is got with a finer topology. In passing, strong algebrability is also obtained. The following elementary lemma is needed.

Lemma 3.4. Let us suppose that $A \subset \mathbb{C}, z_{0} \in A, z_{0}$ is not an isolated point of $A, f: A \rightarrow \mathbb{C}$ is a function that is continuous but not lipchitzian at $z_{0}$, and $g: \Omega \rightarrow \mathbb{C}$ is holomorphic, where $\Omega$ is a domain with $\Omega \supset f(A)$. If $g^{\prime}\left(f\left(z_{0}\right)\right) \neq 0$, then $g \circ f$ is not lipchitzian at $z_{0}$.

Proof. There is a sequence $\left(z_{m}\right)_{m=0}^{\infty} \subset A \backslash\left\{z_{0}\right\}$ such that $\left|f\left(z_{m}\right)-f\left(z_{0}\right)\right| \geq$ $m\left|z_{m}-z_{0}\right|$ for all $m \in \mathbb{N}$. Since $f\left(z_{m}\right) \rightarrow f\left(z_{0}\right)$ as $m \rightarrow \infty$, we get

$$
\lim _{m \rightarrow \infty}\left|\frac{g\left(f\left(z_{m}\right)\right)-g\left(f\left(z_{0}\right)\right)}{f\left(z_{m}\right)-f\left(z_{0}\right)}\right|=\left|g^{\prime}\left(f\left(z_{0}\right)\right)\right|>0 .
$$


Hence, there exists $m_{0} \in \mathbb{N}$ such that

$$
\left|\frac{g\left(f\left(z_{m}\right)\right)-g\left(f\left(z_{0}\right)\right)}{f\left(z_{m}\right)-f\left(z_{0}\right)}\right|>\frac{\left|g^{\prime}\left(f\left(z_{0}\right)\right)\right|}{2}
$$

for all $m \geq m_{0}$. It follows that

$$
\left|(g \circ f)\left(z_{m}\right)-(g \circ f)\left(z_{0}\right)\right| \geq \frac{m\left|g^{\prime}\left(f\left(z_{0}\right)\right)\right|}{2} \cdot\left|z_{m}-z_{0}\right|
$$

for all $m \geq m_{0}$, and we are done.

Theorem 3.5. Assume that $\alpha \in(0,1)$. Then the following holds:

(a) The set $\Lambda_{\alpha} N L_{\mathrm{ae}}(\mathbb{T})$ is strongly c-algebrable.

(b) There exists an infinite dimensional Banach space $\mathcal{B}$ of holomorphic functions in $\mathbb{D}$ such that $\mathcal{B} \backslash\{0\} \subset \Lambda_{\alpha} N L_{\mathrm{ae}}(\mathbb{T})$.

(c) The set $\Lambda_{\alpha} N D_{\mathrm{ae}}(\mathbb{T})$ of all functions $f \in \Lambda_{\alpha}(\mathbb{D})$ such that $f$ is not differentiable a.e. on $\mathbb{T}$ also satisfies the properties in (a) and (b).

Proof. (a) Pick any $f \in \Lambda_{\alpha} N L(\mathbb{T})$ as well as any $\mathfrak{c}$-generated free algebra $\Phi$ consisting, except for zero, of nonconstant entire functions (see Remark 2.5). For every $g \in \Phi \backslash\{0\}$, the set $\left\{w \in \mathbb{C}: g^{\prime}(w)=0\right\}$ has no accumulation points, so the set $S:=\left\{w \in f(\mathbb{T}): g^{\prime}(w)=0\right\}$ is finite. Moreover, $g \circ f \in \Lambda_{\alpha}(\mathbb{D})$ because $g$ is entire.

Let $f^{*}$ denote the radial limit function of $f$, which is defined for each $e^{i \theta} \in \mathbb{T}$ as

$$
f^{*}\left(e^{i \theta}\right)=\lim _{r \rightarrow 1^{-}} f\left(r e^{i \theta}\right) .
$$

Since $f \in A(\mathbb{D}) \subset H^{1}$ (the Hardy space of order 1 on the disc), it is known that, given $w \in \mathbb{C}$, the set $\left\{e^{i \theta} \in \mathbb{T}: f^{*}\left(e^{i \theta}\right)=w\right\}$ has null measure (see $\left[44\right.$, p. 345]). Since $f$ is continuous on $\overline{\mathbb{D}}$, it follows that $f^{*}=f$ on $\mathbb{T}$, so each set $\{z \in \mathbb{T}: f(z)=w\}$ has null measure. Hence the set

$$
E_{f}:=\bigcup_{w \in S}\{z \in \mathbb{T}: f(z)=w\}
$$

has null measure and $g^{\prime}\left(f\left(z_{0}\right)\right) \neq 0$ for every $z_{0} \in \mathbb{T} \backslash E_{f}$. According to Lemma 3.4 (with $A=\mathbb{T}$ and $\Omega=\mathbb{C}$ ), the function $g \circ f$ is not lipschitzian at any $z_{0} \in \mathbb{T} \backslash E_{f}$. This shows that $g \circ f \in \Lambda N L_{\text {ae }}(\mathbb{T})$. Then Lemma 2.4 (with $\mathcal{F}=\Lambda_{\alpha} N L_{\mathrm{ae}}(\mathbb{T})$ ) yields the conclusion.

(b) Let us consider the function $v(z)=e^{-|z|}$, as well as the weighted space of entire functions associated to $v$ defined as

$$
H_{v}(\mathbb{C}):=\left\{g \in H(\mathbb{C}): \sup _{z \in \mathbb{C}} v(z)|g(z)|<\infty\right\},
$$

that is a Banach space under the norm $\|g\|_{v}:=\sup _{z \in \mathbb{C}}(v(z)|g(z)|)$ (see, e.g., [38]). Convergence in this norm implies uniform convergence on compacta. We pick any $f \in \Lambda_{\alpha} N L(\mathbb{T})$ and define the vector space

$$
\mathcal{B}:=\left\{g \circ f: g \in H_{v}(\mathbb{C}) \text { and } g(0)=0\right\} .
$$


If $g \circ f \in \mathcal{B} \backslash\{0\}$ with $g \in H_{v}(\mathbb{C})$ and $g(0)=0$, then the function $g$ cannot be constant and, as in (a), we get that $g \circ f \in \Lambda_{\alpha} N L_{\mathrm{ae}}(\mathbb{T})$. That is, $\mathcal{B} \backslash\{0\} \subset \Lambda_{\alpha} N L_{\text {ae }}(\mathbb{T})$. Moreover, it follows from the identity principle for analytic functions that every function $h \in \mathcal{B}$ has a unique representation $h=g \circ f$ with $g \in H_{v}(\mathbb{C})$ and $g(0)=0$. It is also obtained that the functions $\left\{f^{k}: k \in \mathbb{N}\right\}$ form a linearly independent subset of $\mathcal{B}$ (note that the monomials $z^{k}$ are in $H_{v}(\mathbb{C})$ ). Finally, it is easy to see that the formula $\|h\|=\|g\|_{v}$ provides $\mathcal{B}$ with a norm, that is complete because $\|\cdot\|_{v}$ is complete on $H_{v}(\mathbb{C})$.

(c) This follows from (a) and (b) because $\Lambda_{\alpha} N L_{\mathrm{ae}}(\mathbb{T}) \subset \Lambda_{\alpha} N D_{\mathrm{ae}}(\mathbb{T})$.

A natural problem is to study the size of the intersection of all classes $\Lambda_{\alpha} N L(\mathbb{T})$. We are going to show that its size is large, at least in the topological sense (see Proposition 3.6 below). For this, we consider the vector space

$$
\Lambda(\mathbb{D}):=\bigcap_{0<\alpha<1} \Lambda_{\alpha}(\mathbb{D})
$$

and its subset of functions that are not lipschitzian at any point of $\mathbb{T}$ :

$$
\Lambda N L(\mathbb{T}):=\bigcap_{0<\alpha<1} \Lambda_{\alpha} N L(\mathbb{T})
$$

Note that thanks to the inclusions $(2.2)$ we get

$$
\Lambda(\mathbb{D})=\bigcap_{0<\alpha<1} \Lambda^{\alpha}(\mathbb{D})=\bigcap_{n \in \mathbb{N}} \Lambda^{\frac{n}{n+1}}(\mathbb{D})=\bigcap_{n \in \mathbb{N}} \Lambda_{\frac{n}{n+1}}(\mathbb{D}) .
$$

For each $n \in \mathbb{N}$, let $X_{n}:=\Lambda_{\frac{n}{n+1}}(\mathbb{D})$. Since the sequence $\left\{X_{n}\right\}_{n \geq 1}$ is decreasing and each inclusion $X_{n+1} \hookrightarrow X_{n}$ is linear and continuous, the natural topology on $\Lambda(\mathbb{D})=\bigcap_{n \geq 1} X_{n}$ appears to be the topology $\tau_{\pi}$ of the projective limit of the sequence of Banach spaces $\left\{\left(X_{n},\|\cdot\|_{\frac{n}{n+1}}\right)\right\}_{n \geq 1}$ (see, e.g., [33, Section 11, especially pp. 154-155]). Since every $X_{n}^{n+1}$ is a separable Banach space, we have that $\tau_{\pi}$ makes $\Lambda(\mathbb{D})$ a separable Fréchet space, that is, a separable metrizable complete locally convex space. In fact, the restrictions to $\Lambda(\mathbb{D})$ of the norms $\|\cdot\|_{\frac{n}{n+1}}(n=1,2, \ldots)$ generate the topology of $\tau_{\pi}$. Notice that we have the Baire category theorem at our disposal.

Proposition 3.6. The set $\Lambda N L(\mathbb{T})$ is a residual subset of $\Lambda(\mathbb{D})$.

Proof. For a continuous function $h: \overline{\mathbb{D}} \rightarrow \mathbb{C}$, we will set

$$
\|h\|_{\infty}:=\sup _{z \in \overline{\mathbb{D}}}|h(z)| \text {. }
$$

A basis of the topology of $\Lambda(\mathbb{D})$ is formed by all the sets

$$
\left\{g \in \Lambda(\mathbb{D}):\|g-f\|_{\frac{n}{n+1}}<\varepsilon, n \in\{1, \ldots, N\}\right\},
$$

where $f \in \Lambda(\mathbb{D}), \varepsilon>0$ and $N \in \mathbb{N}$. But since the set $\mathcal{P}$ of polynomials is dense in $\Lambda(\mathbb{D})$ (because $\mathcal{P}$ is dense in each $\Lambda_{\frac{n}{n+1}}$ ) and $\|\cdot\|_{\frac{n}{n+1}} \leq\|\cdot\|_{\frac{m}{m+1}}$ 
whenever $n \leq m$, we can take the family

$$
\{V(P, \varepsilon, n): P \in \mathcal{P}, \varepsilon>0, n \in \mathbb{N}\}
$$

as a basis, where

$$
V(P, \varepsilon, n):=\left\{g \in \Lambda N L(\mathbb{T}):\|g-P\|_{\frac{n}{n+1}}<\varepsilon\right\} .
$$

Observe first that we can write

$$
\Lambda(\mathbb{D}) \backslash \Lambda N L(\mathbb{T})=\bigcup_{m, p=1}^{\infty} Y_{m, p}
$$

where

$$
\begin{aligned}
Y_{m, p}= & \left\{f \in \Lambda(\mathbb{D}): \exists z_{0} \in \mathbb{T}\right. \text { such that } \\
& \left.\left|f(z)-f\left(z_{0}\right)\right| \leq m\left|z-z_{0}\right| \text { if } z \in \mathbb{T} \text { and }\left|z-z_{0}\right|<1 / p\right\} .
\end{aligned}
$$

We will prove that each $Y_{m, p}$ is closed in $\Lambda(\mathbb{D})$. Let $\left(f_{i}\right)_{i=1}^{\infty}$ be a sequence in $Y_{m, p}$ that converges to $f \in \Lambda(\mathbb{D})$. In particular, $f_{i} \rightarrow f$ uniformly on $\overline{\mathbb{D}}$. For every $i$ there exists $z_{i} \in \mathbb{T}$ such that $\left|f(z)-f\left(z_{i}\right)\right| \leq m\left|z-z_{i}\right|$ if $z \in \mathbb{T}$ and $\left|z-z_{i}\right|<1 / p$. We can assume that $\left(z_{i}\right)_{i=1}^{\infty}$ converges to $z_{0} \in \mathbb{T}$. Given $z \in \mathbb{T}$ such that $\left|z-z_{0}\right|<1 / p$, there is $i_{0}$ such that $\left|z-z_{i}\right|<1 / p$ for all $i \geq i_{0}$. If $i \geq i_{0}$, then

$$
\begin{aligned}
\left|f(z)-f\left(z_{0}\right)\right| \leq & \left|f(z)-f_{i}(z)\right|+\left|f_{i}(z)-f_{i}\left(z_{i}\right)\right|+ \\
& +\left|f_{i}\left(z_{i}\right)-f\left(z_{i}\right)\right|+\left|f\left(z_{i}\right)-f\left(z_{0}\right)\right| \\
\leq & 2|| f_{i}-f \|_{\infty}+m\left|z-z_{i}\right|+\left|f\left(z_{i}\right)-f\left(z_{0}\right)\right| \rightarrow m\left|z-z_{0}\right|
\end{aligned}
$$

Hence, $\left|f(z)-f\left(z_{0}\right)\right| \leq m\left|z-z_{0}\right|$, which shows that $Y_{m, p}$ is closed in $\Lambda(\mathbb{D})$. To sum up, we have obtained that $\Lambda(\mathbb{D}) \backslash \Lambda N L(\mathbb{T})$ is an $\mathrm{F}_{\sigma}$ set.

According to the Baire category theorem, it suffices to show that each set $Y_{m, p}$ has empty interior. With this aim, fix $(m, p, n) \in \mathbb{N}^{3}, \varepsilon>0$ and $P \in \mathcal{P}$. It is enough to exhibit a function belonging to $V(P, \varepsilon, n) \backslash Y_{m, p}$. For every entire function $g$ and every pair $(z, w) \in \overline{\mathbb{D}}$ we have

$$
g(z)-g(w)=\int_{[w, z]} g^{\prime}(\xi) d \xi
$$

Then we derive that $|g(z)-g(z)| \leq\left\|g^{\prime}\right\|_{\infty}|z-w|$. In particular, we get that

$$
\sup _{\substack{z, w \in \mathbb{D} \\ z \neq w}}\left|\frac{z^{k}-w^{k}}{z-w}\right| \leq k
$$

for all $k \in \mathbb{N}$ and also $|P(z)-P(w)| \leq\left\|P^{\prime}\right\|_{\infty}|z-w|$ for all $z, w \in \mathbb{T}$. Choose an $k \in \mathbb{N}$ with

$$
k>\frac{4^{n+1} \cdot 2\left(m+\left\|P^{\prime}\right\|_{\infty}\right)^{n+1}}{\varepsilon^{n+1}}
$$

and define

$$
\lambda:=\frac{\varepsilon}{2 k^{\frac{n}{n+1}} 2^{\frac{1}{n+1}}} \quad \text { and } \quad f(z):=P(z)+\lambda z^{k} \in \mathcal{P} \subset \Lambda(\mathbb{D}) .
$$


We obtain

$$
\begin{aligned}
\|f-P\|_{\frac{n}{n+1}} & =\left\|\lambda z^{k}\right\|_{\frac{n}{n+1}}=\lambda \sup _{\substack{z, w \in \mathbb{D} \\
z \neq w}} \frac{\left|z^{k}-w^{k}\right|}{|z-w|^{\frac{n}{n+1}}} \\
& =\lambda \sup _{\substack{z, w \in \mathbb{D} \\
z \neq w}}\left(\left|\frac{z^{k}-w^{k}}{z-w}\right|^{\frac{n}{n+1}} \cdot\left|z^{k}-w^{k}\right|^{\frac{1}{n+1}}\right) .
\end{aligned}
$$

By (3.7),

$$
\|f-P\|_{\frac{n}{n+1}} \leq \frac{\varepsilon}{2 k^{\frac{n}{n+1}} 2^{\frac{1}{n+1}}} \cdot k^{\frac{n}{n+1}} \cdot \sup _{\substack{z, w \in \mathbb{D} \\ z \neq w}}\left|z^{k}-w^{k}\right|^{\frac{1}{n+1}}=\frac{\varepsilon}{2}<\varepsilon .
$$

Hence $f \in V(P, \varepsilon, n)$. Finally, fix $z_{0} \in \mathbb{T}$. Since the modulus of the derivative of $z^{k}$ at $z_{0}$ is $k$, we have that

$$
\left|\frac{z^{k}-z_{0}^{k}}{z-z_{0}}\right|>\frac{k}{2}
$$

whenever $z$ is close enough to $z_{0}$. If we choose such a $z \in \mathbb{T}$ with $\left|z-z_{0}\right|<$ $1 / p$, then

$$
\begin{aligned}
\left|f(z)-f\left(z_{0}\right)\right| & \geq \lambda\left|z^{k}-z_{0}{ }^{k}\right|-\left|P(z)-P\left(z_{0}\right)\right| \\
& \geq \frac{\lambda k}{2}\left|z-z_{0}\right|-\left\|P^{\prime}\right\|_{\infty}\left|z-z_{0}\right| \\
& =\left(\frac{\varepsilon k}{4 k^{\frac{n}{n+1}} 2^{\frac{1}{n+1}}}-\left\|P^{\prime}\right\|_{\infty}\right)\left|z-z_{0}\right| \\
& >\left(m+\left\|P^{\prime}\right\|_{\infty}-\left\|P^{\prime}\right\|_{\infty}\right)\left|z-z_{0}\right|=m\left|z-z_{0}\right| .
\end{aligned}
$$

In other words, $f \notin Y_{m, p}$, as required. Thus, we have proved that each $Y_{m, p}$ is closed and has empty interior and, consequently, the set $\Lambda N L(\mathbb{T})$ is residual in $\Lambda(\mathbb{D})$.

In view of the previous results, the following questions arise naturally:

(a) Is $\Lambda_{\alpha} N L(\mathbb{T})$ spaceable in $\Lambda_{\alpha}(\mathbb{D})$ ? Is it (strongly) algebrable?

(b) What lineability properties does the family $\Lambda N L(\mathbb{T})$ enjoy?

Remark 3.7. As a related result, given any $\alpha \in(0,1)$, we may assert spaceability in $\Lambda_{\alpha}(\mathbb{D})$ for the family

$$
\mathcal{F}:=\Lambda_{\alpha}(\mathbb{D}) \backslash \bigcup_{\alpha<\beta \leq 1} \Lambda_{\beta}(\mathbb{D}) .
$$

For this, we can invoke a theorem due to Kitson and Timoney [36, Theorem 3.3] asserting that if $\left\{Z_{n}\right\}_{n=1}^{\infty}$ is a sequence of Banach spaces, $X$ is a Fréchet space and $T_{n}: Z_{n} \rightarrow X$ are continuous linear mappings such that $Y:=\operatorname{span}\left(\bigcup_{n \in \mathbb{N}} T_{n}\left(Z_{n}\right)\right)$ is not closed in $X$, then the complement $X \backslash Y$ is spaceable in $X$. Let $X:=\Lambda_{\alpha}(\mathbb{D}), \alpha(n):=\alpha+\frac{1-\alpha}{n}, Z_{n}:=\Lambda^{\alpha(n)}(\mathbb{D})$ and 
$T_{n}$ be the natural inclusion $Z_{n} \hookrightarrow X$. Note that by the inclusions (2.2) we have that

$$
Y:=\bigcup_{\alpha<\beta \leq 1} \Lambda_{\beta}(\mathbb{D})=\bigcup_{n \in \mathbb{N}} \Lambda^{\alpha(n)}(\mathbb{D})=\operatorname{span}\left(\bigcup_{n \in \mathbb{N}} T_{n}\left(\Lambda^{\alpha(n)}(\mathbb{D})\right)\right) .
$$

On the one hand, the set $Y$ is dense in $X$ because $\mathcal{P} \subset Y$. On the other hand, $Y$ is not closed in $X$ because, otherwise, we would have $Y=X$, which is not possible because $X$ is a Baire space and $Y$ is a set of first category in $X$. Indeed, we have that $Y=\bigcup_{n, m=1}^{\infty} Y_{n, m}$, where

$$
Y_{n, m}:=\left\{f \in \Lambda_{\alpha}(\mathbb{D}):|f(z)-f(w)| \leq m|z-w|^{\alpha(n)} \text { for all } z, w \in \mathbb{D}\right\},
$$

and similarly (but in an easier way) to the proof of Proposition 3.6, one can see that each $Y_{n, m}$ is a closed subset in $\Lambda_{\alpha}(\mathbb{D})$ with empty interior. Consequently, the mentioned theorem by Kitson and Timoney applies, so $\mathcal{F}=X \backslash Y$ is spaceable in $\Lambda_{\alpha}(\mathbb{D})$.

\section{Non-Preservation of Borel Sets and Cantor boundary BEHAVIOR ON THE UNIT CIRCLE}

Recall that the $\sigma$-algebra $\mathfrak{B}$ of Borel sets in the one-point compactification $\mathbb{C}_{\infty}=\mathbb{C} \cup\{\infty\}$ of $\mathbb{C}$ is defined as the smallest $\sigma$-algebra that contains all open sets. It is well known that the preimage of a Borel set under a continuous mapping is again a Borel set, whereas the image of a Borel set need not to be a Borel set. Given a subset $A \subset \mathbb{C}$, we say that a complex-valued mapping $f$ preserves Borel sets on $A$ if $f$ is defined on $A$ and the following property holds:

$$
B \subset A, B \in \mathfrak{B} \Longrightarrow f(B) \in \mathfrak{B} .
$$

As a consequence of the Lusin-Purves Theorem [19], a function $f \in A(\mathbb{D})$ preserves Borel sets on $\mathbb{T}$ if and only if the set

$$
\{w \in \mathbb{C}: w=f(z) \text { for uncountably many } z \in \mathbb{T}\}
$$

is countable. If $f$ is a complex-valued function defined on $\mathbb{T}$, then it is said that the image of the unit circle under $f$ is a Peano curve if it covers a nonempty open subset of the plane.

Let $\mathcal{F}_{\mathrm{O}, \infty, \mathbb{T}}$ be the family of all functions $f \in A(\mathbb{D})$ assuming every value of some open set - depending on $f$ - uncountably many times on $\mathbb{T}$. As a consequence of Theorem $\mathrm{A}$ in [8], if $a>1$ and $b \in\{2,3,4 \ldots\}$, then the function

$$
f_{a, b}(z):=\sum_{n=1}^{\infty} \frac{z^{b^{n}}}{n^{a}}
$$

belongs to $\mathcal{F}_{\mathrm{O}, \infty, \mathbb{T}}$.

Theorem 4.1. The set $\mathcal{F}_{\mathrm{o}, \infty, \mathbb{T}}$ is strongly $\mathfrak{c}$-algebrable. 
Proof. Take a function $f:=f_{a, b}$ as above. Then there is an open set $U \subset \mathbb{C}$ such that $f$ assumes every point of $U$ uncountable many times on $\mathbb{T}$. If $\varphi$ is any nonconstant entire function, then $\varphi(U)$ is also open and $\varphi \circ f$ assumes every point of $\varphi(U)$ uncountable many times on $\mathbb{T}$. That is, $\varphi \circ f \in \mathcal{F}_{\mathrm{o}, \infty, \mathbb{T}}$ for every nonconstant entire function $\varphi$. Finally, it suffices to apply Lemma 2.4 with $\Omega=\overline{\mathbb{D}}, \mathcal{F}=\mathcal{F}_{\mathrm{O}, \infty, \mathbb{T}}$ and $\Phi$ any of the free algebras given in Remark 2.5 .

Corollary 4.2. The set of functions $f \in A(\mathbb{D})$ such that $f(\mathbb{T})$ is a Peano curve is strongly $\mathfrak{c}$-algebrable.

Remarks 4.3. 1. As a consequence of Theorem 4.8 in [15], the family of continuous functions $f:[0,1] \rightarrow \mathbb{C}$ such that $f([0,1])$ covers a nonempty open set of $\mathbb{C}$ is strongly $\aleph_{0}$-algebrable, where $\aleph_{0}=\operatorname{card}(\mathbb{N})$. Then this result is strengthened by Corollary 4.2 .

2. Other examples of $A(\mathbb{D})$-functions such that $f(\mathbb{T})$ is a Peano curve can be found in [45].

Corollary 4.4. The set of functions in $f \in A(\mathbb{D})$ such that $f$ does not preserve Borel sets on the unit circle is strongly $\mathfrak{c}$-algebrable.

Let us now consider for a function $f \in A(\mathbb{D})$ the decomposition

$$
\mathbb{C}_{\infty} \backslash f(\mathbb{T})=\bigcup_{j \geq 1} W_{j},
$$

where the $W_{j}$ are connected components. Let $\partial f(\mathbb{D})$ and $\partial W_{j}$ denote the $\mathbb{C}_{\infty}$-boundary of $f(\mathbb{D})$ and $W_{j}$, respectively. According to Dong, Lau and Liu [21, Definition 1.1] (see also [37]), a function $f \in A(\mathbb{D})$ is said to have the Cantor boundary behavior on $\mathbb{D}$ if $f^{-1}(\partial f(\mathbb{D}))$ and all $f^{-1}\left(\partial W_{j}\right)$ are Cantor-type sets, that is, each of these pre-images is uncountable, nowhere dense and closed in $\mathbb{T}$.

A remarkable result due to Dong et al. [21, Theorem 5.3] asserts that if $f \in A(\mathbb{D})$ is nonconstant and the set of limit points of $\left\{z \in \mathbb{D}: f^{\prime}(z)=0\right\}$ equals $\mathbb{T}$, then $f$ has the Cantor boundary behavior on $\mathbb{D}$. As an example, the function

$$
f_{q, \beta}(z):=\sum_{n=1}^{\infty} q^{-\beta n} z^{q^{n}},
$$

where $0<\beta<1$ and $q \geq 2$ is an integer, satisfies that the set of limit points of $\left\{z \in \mathbb{D}: f_{p, \beta}^{\prime}(z)=0\right\}$ equals $\mathbb{T}[21$, Corollary 6.5].

Moreover if $g$ is an analytic function defined on a domain $\Omega \supset \overline{f(\mathbb{D})}$ and $f$ is a function in $A(\mathbb{D})$ such that the set of limit points of $\left\{z \in \mathbb{D}: f^{\prime}(z)=0\right\}$ equals $\mathbb{T}$, then the chain rule implies that $g \circ f$ shares the same property. This allows us to construct a large algebras of functions satisfying it: just fix a function $f_{q, \beta}$ as above and apply Lemma 2.4 together with Remark 2.5. This yields the following result, which puts the end on this paper. 
Theorem 4.5. The set of functions in $A(\mathbb{D})$ such that the set of limit points of $\left\{z \in \mathbb{D}: f^{\prime}(z)=0\right\}$ equals $\mathbb{T}$ is strongly $\mathfrak{c}$-algebrable. Hence the set of functions in $A(\mathbb{D})$ having the Cantor boundary behavior on $\mathbb{D}$ is strongly $\mathfrak{c}$-algebrable.

Acknowledgements. The first author was supported by the Plan Andaluz de Investigación de la Junta de Andalucía FQM-127 Grant P08-FQM-03543 and by MEC Grant MTM2015-65242-C2-1-P. The second author was supported by MINECO MTM2016-75963-P. The third and fourth authors were supported by the Spanish Ministry of Economy, Grant MTM2015-65825-P.

\section{REFERENCES}

[1] N. Albuquerque, L. Bernal-González, D. Pellegrino, and J. B. Seoane-Sepúlveda, Peano curves on topological vector spaces, Linear Algebra Appl. 460 (2014), 81-96.

[2] R. Aron, L. Bernal-González, D. Pellegrino, and J. B. Seoane-Sepúlveda, Lineability: The search for linearity in Mathematics, Monographs and Research Notes in Mathematics, Chapman \& Hall/CRC, Boca Raton, FL, 2016.

[3] R. M. Aron, F. J. García-Pacheco, D. Pérez-García, and J. B. Seoane-Sepúlveda, On dense-lineability of sets of functions on $\mathbb{R}$, Topology 48 (2009), no. 2-4, 149-156.

[4] R. M. Aron, V. I. Gurariy, and J. B. Seoane-Sepúlveda, Lineability and spaceability of sets of functions on $\mathbb{R}$, Proc. Amer. Math. Soc. 133 (2005), no. 3, 795-803.

[5] R. M. Aron, D. Pérez-García, and J. B. Seoane-Sepúlveda, Algebrability of the set of nonconvergent Fourier series, Studia Math. 175 (2006), no. 1, 83-90.

[6] M. Balcerzak, A. Bartoszewicz, and M. Filipczak, Nonseparable spaceability and strong algebrability of sets of continuous singular functions, J. Math. Anal. Appl. 407 (2013), no. 2, 263-269.

[7] S. Banach, Über die Bairesche Kategorie gewisser Funktionenmengen, Studia Math. 3 (1931), 174-179.

[8] K. Baranski, On some lacunary power series, Michigan Math. J. 54 (2006), no. 1, $65-79$.

[9] A. Bartoszewicz and S. Głąb, Large function algebras with certain topological properties, J. Funct. Spaces 2015, Article ID 761924, 7 pages.

[10] F. Bastin, J. A. Conejero, C. Esser, and J. B. Seoane-Sepúlveda, Algebrability and nowhere Gevrey differentiability, Israel J. Math. 205 (2015), no. 1, 127-143.

[11] F. Bayart and L. Quarta, Algebras in sets of queer functions, Israel J. Math. 158 (2007), no. 1, 285-296.

[12] L. Bernal-González, Dense-lineability in spaces of continuous functions, Proc. Amer. Math. Soc. 136 (2008), no. 9, 3163-3169.

[13] Vector spaces of non-extendable holomorphic functions, J. Anal. Math. 134 (2018), 769-786.

[14] L. Bernal-González, A. Bonilla, J. López-Salazar, and J. B. Seoane-Sepúlveda, Nowhere hölderian functions and Pringsheim singular functions in the disc algebra, Monatsh Math. (2018).

[15] L. Bernal-González, M. C. Calderón-Moreno, and J. A. Prado-Bassas, The set of space-filling curves: topological and algebraic structures, Linear Algebra Appl. 467 (2015), 57-74.

[16] L. Bernal-González, J. López-Salazar, and J. B. Seoane-Sepúlveda, On Weierstrass' monsters in the disc algebra, Bull. Belg. Math. Soc. (2018), in press.

[17] L. Bernal-González and M. Ordóñez Cabrera, Lineability criteria, with applications, J. Funct. Anal. 266 (2014), no. 6, 3997-4025. 
[18] L. Bernal-González, D. Pellegrino, and J. B. Seoane-Sepúlveda, Linear subsets of nonlinear sets in topological vector spaces, Bull. Amer. Math. Soc. (N.S.) 51 (2014), no. $1,71-130$.

[19] A. Cantón, A. Granados, and Ch. Pommerenke, Borel images and analytic functions, Michigan Math. J. 52 (2004), no. 2, 279-287.

[20] D. Cariello and J. B. Seoane-Sepúlveda, Basic sequences and spaceability in $\ell_{p}$ spaces, J. Funct. Anal. 266 (2014), no. 6, 3797-3814.

[21] X. H. Dong, K. S. Lau, and J. C. Liu, Cantor boundary behavior of analytic functions, Adv. Math. 232 (2013), 543-570.

[22] P. L. Duren, Theory of $H^{p}$ spaces, Dover, Mineola, New York, 2000.

[23] P. H. Enflo, V. I. Gurariy, and J. B. Seoane-Sepúlveda, Some results and open questions on spaceability in function spaces, Trans. Amer. Math. Soc. 366 (2014), no. 2, $611-625$.

[24] A. Eskenazis, Topological genericity of nowhere differentiable functions in the disc algebra, Arch. Math. (Basel) 103 (2014), no. 1, 85-92.

[25] A. Eskenazis and K. Makridis, Topological genericity of nowhere differentiable functions in the disc and polydisc algebras, J. Math. Anal. Appl. 420 (2014), no. 1, 435446.

[26] V. P. Fonf, V. I. Gurariy, and M. I. Kadets, An infinite dimensional subspace of $C[0,1]$ consisting of nowhere differentiable functions, C. R. Acad. Bulgare Sci. 52 (1999), no. 11-12, 13-16.

[27] J. L. Gámez, G. A. Muñoz-Fernández, and J. B. Seoane-Sepúlveda, Lineability and additivity in $\mathbb{R}^{\mathbb{R}}$, J. Math. Anal. Appl. 369 (2010), no. 1, 265-272.

[28] J. L. Gámez-Merino and J. B. Seoane-Sepúlveda, An undecidable case of lineability in $\mathbb{R}^{\mathbb{R}}$, J. Math. Anal. Appl. 401 (2013), no. 2, 959-962.

[29] D. García, B. C. Grecu, M. Maestre, and J. B. Seoane-Sepúlveda, Infinite dimensional Banach spaces of functions with nonlinear properties, Math. Nachr. 283 (2010), no. 5, $712-720$.

[30] D. Girela and C. González, Some results on mean Lipschitz spaces of analytic functions, Rocky Mt. J. Math. 30 (2000), no. 3, 901-922.

[31] V. I. Gurariŭ, Linear spaces composed of everywhere nondifferentiable functions, C. R. Acad. Bulgare Sci. 44 (1991), no. 5, 13-16 (in Russian).

[32] S. Hencl, Isometrical embeddings of separable Banach spaces into the set of nowhere approximatively differentiable and nowhere Hölder functions, Proc. Amer. Math. Soc. 128 (2000), no. 12, 3505-3511.

[33] J. Horváth, Topological Vector Spaces and Distributions (Vol. I), Addison-Wesley, Reading, MA, 1966.

[34] P. Jiménez-Rodríguez, G. A. Muñoz-Fernández, and J. B. Seoane-Sepúlveda, On Weierstrass' Monsters and lineability, Bull. Belg. Math. Soc. Simon Stevin 20 (2013), no. 4, 577-586.

[35] K. Kavvadias and K. Makridis, Nowhere differentiable functions with respect to the position, arXiv:1701.04875v1 [math.CV], Preprint (2017).

[36] D. Kitson and R. M. Timoney, Operator ranges and spaceability, J. Math. Anal. Appl. 378 (2011), no. 2, 680-686.

[37] J. C. Liu, X. H. Dong, and S. M. Pen, A note on Cantor boundary behavior, J. Math. Anal. Appl. 408 (2013), 795-801.

[38] W. Lusky, On weighted spaces of harmonic and holomorphic functions, J. London Math. Soc. 51 (1995), 309-320.

[39] S. Mazurkiewicz, Sur les fonctions non dérivables, Studia Math. 3 (1931), 92-94.

[40] A. G. O'Farrell, Hausdorff content and rational approximation in fractional Lipschitz norms, Trans. Amer. Math. Soc. 228 (1977), 187-206.

[41] J. C. Oxtoby, Measure and Category, 2nd edition, Springer-Verlag, New York, 1980. 
[42] M. Pavlovic, Function Classes on the Unit Disc. An Introduction, Walter de Gruyter, Berlin, 2014.

[43] L. Rodríguez-Piazza, Every separable Banach space is isometric to a space of continuous nowhere differentiable functions, Proc. Amer. Math. Soc. 123 (1995), no. 12, 3649-3654.

[44] W. Rudin, Real and complex analysis, 3rd ed., McGraw-Hill Book Co., New York, 1987.

[45] H. Sagan, Space-filling Curves, Universitext, Springer-Verlag, New York, 1994.

[46] J. B. Seoane-Sepúlveda, Chaos and lineability of pathological phenomena in analysis, ProQuest LLC, Ann Arbor, MI, 2006. Thesis (Ph.D.)-Kent State University.

[47] J. Thim, Continuous nowhere differentiable functions, Luleå University of Technology, 2003. Master Thesis.

[48] A. C. M. Van Rooij and W. H. Schikhof, A Second Course on Real Functions, Cambridge University Press, Cambridge, 1982.

Departamento de Análisis Matemático, Facultad de Matemáticas Instituto de Matemáticas Antonio de Castro Brzezicki (IMUS)

UnivERSIDAD DE SEVILla

Avenida Reina Mercedes, Sevilla, 41080, Spain

E-mail address: lbernal@us.es

Departamento de Análisis Matemático

UNIVERSIDAD DE LA LAGUNA

La Laguna (Tenerife), 38271, Spain

E-mail address: abonilla@ull.es

Departamento de Matemática Aplicada a las Tecnologías

DE LA INFORMACIÓN Y LAS COMUNICACIONES

Escuela Técnica Superior de Ingeniería y Sistemas de Telecomunicación

Universidad Politécnica de MAdRID

Carretera de Valencia, Km. 7.

MADRID, 28031, SPAIN

E-mail address: jeronimo.lopezsalazar@upm.es

Instituto DE MATEMÁtica Interdisciplinar (IMI)

Departamento de Análisis y Matemática Aplicada,

Facultad de Ciencias Matemáticas

Universidad Complutense de Madrid

Plaza de Ciencias 3

MADRID, 28040, SPAIN

E-mail address: jseoane@ucm.es 